\title{
Microbial metabolism inferred from chemical and isotopic compositions of pore water around bananas discovered on the deep-sea floor in the Tenryu Submarine Canyon
}

\author{
Tomohiro Toki ${ }^{1 a^{*}}$, Kiichiro Kawamura ${ }^{2 \mathrm{~b}}$, Urumu Tsunogai ${ }^{3 \mathrm{c}}$, and Toshitaka Gamo ${ }^{1 \mathrm{~d}}$
}

We describe in detail the pore water chemistry in the sediments below a white mat around a bunch of bananas at a water depth of 2,200 $\mathrm{m}$ in the Tenryu Submarine Canyon. We infer the metabolism of microbes in the sediments around the bananas based on the chemical and isotopic compositions of the pore water. On the basis of the relation between ammonia and total carbonate $\left(\Sigma \mathrm{CO}_{2}\right)$ concentrations in the pore water, we identified that an excess $\Sigma \mathrm{CO}_{2}$ was distributed around the bananas that cannot be explained by the decomposition of organic matter derived from marine organisms, indicating that the bananas decomposed to generate the excess $\Sigma \mathrm{CO}_{2}$. We conclude that the bananas built a local organic-rich environment, stimulating the activity of organotrophic bacteria.

Keywords : deep-sea floor, banana, pore water, chemical composition, microbial metabolism

Received 25 January 2017 ; Revised 9 May 2017 ; Accepted 15 May 2017

1 Marine Inorganic Chemistry Group, Department of Chemical Oceanography, Ocean Research Institute, The University of Tokyo

2 Fukada Geological Institute

3 Earth and Planetary System Science, Faculty of Science, Hokkaido University

Present affiliation:

a Department of Chemistry, Biology and Marine Science, Faculty of Science, University of the Ryukyus

b Graduate School of Science and Engineering, Yamaguchi University

c Graduate School of Environmental Studies, Nagoya University

d Marine Inorganic Chemistry, Department of Chemical Oceanography, Atmosphere and Ocean Research Institute, The University of Tokyo

*Corresponding author:

Tomohiro Toki

Department of Chemistry, Biology and Marine Science, Faculty of Science, University of the Ryukyus

1 Senbaru, Nishihara, Nakagami, Okinawa 903-0213, Japan

toki@sci.u-ryukyu.ac.jp

Copyright by Japan Agency for Marine-Earth Science and Technology 


\section{Introduction}

Organic matter produced at the surface of the ocean is primarily decomposed prior to arriving on the deep-sea floors, and biomasses at this deep level are extremely low relative to that of the surface ocean (Gage and Tyler, 1991); however, in hydrothermal systems and cold seep areas, tremendous high-density biomasses have been discovered (Lonsdale, 1977; Suess et al., 1985). These biological communities comprise ecosystems furnished by chemosynthetic microbes utilizing hydrogen sulfide or hydrocarbons in hydrothermal or cold seeping fluid, respectively, as their energy sources (Sibuet and Olu, 1998; Tunnicliffe et al., 1998; Van Dover, 2000). Moreover, the whale fall or cargo of shipwrecked vessels have been reported as energy sources of benthic communities on the deep-sea floor (Smith and Baco, 2003; Dando et al., 1992). Metabolisms of these benthic communities have been studied very well (e.g., Smith et al., 2015), but interstitial water around these communities have rarely been studied.

Vertical profiles of chemical components in interstitial water provide us various information; fluid migration including methane-hydrate chronicle from conservative components (e.g., Toki et al., 2017), metabolism including microbially-unknown processes

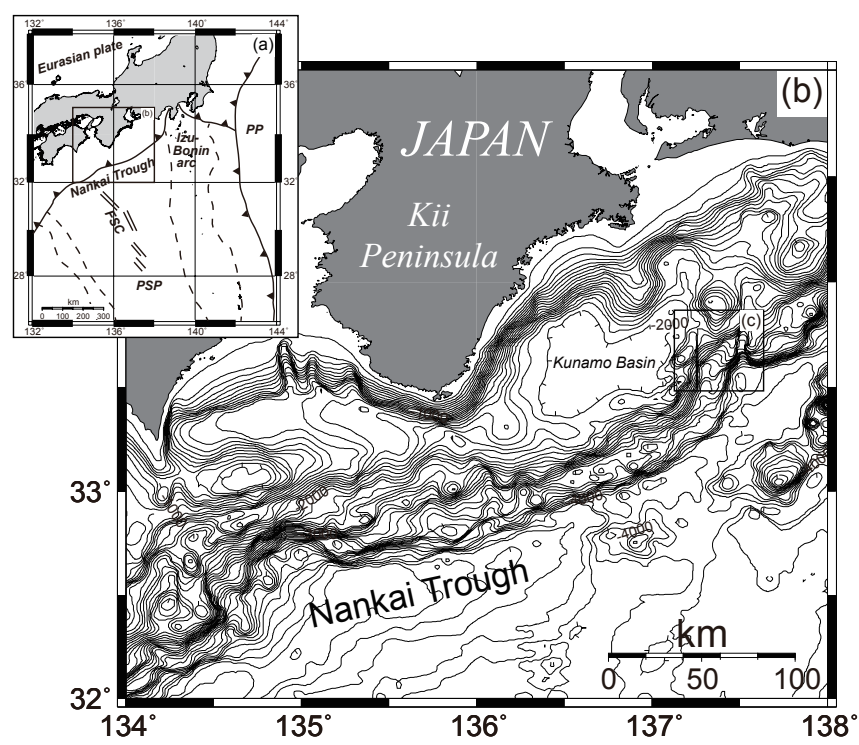

from biochemical components (e.g., Martens and Berner, 1974), or formation process of authigenic and foraminiferal carbonates from various isotopic compositions (Gieskes et al., 2005; Gieskes et al., 2011). In this present study, we sampled the pore water around a bunch of bananas discovered during a dive survey in the deep ocean, clarifying a peculiar microbial metabolism around the bananas via geochemical interpretation.

\section{Geological settings}

The Tenryu Submarine Canyon is part of the great erosional valley on the seafloor located in the eastern Nankai Trough (Fig. 1b). The Nankai Trough is a plate boundary between the Philippine Sea Plate and the Eurasia Plate (Fig. 1a), and an accretionary prism has been forming on the landward plate by accreted sediments scraped off from the subducting Philippine Sea Plate (Ranken et al., 1984; Seno, 1977). The Tenryu Submarine Canyon cuts deeply across the Nankai accretionary prism, and the ongoing accretionary processes of the accretionary prism can be described by direct observations during submersible surveys along the walls of the Tenryu canyon in exact detail (Kawamura et al., 2009). Three major faults, the Kodaiba Fault, Tokai

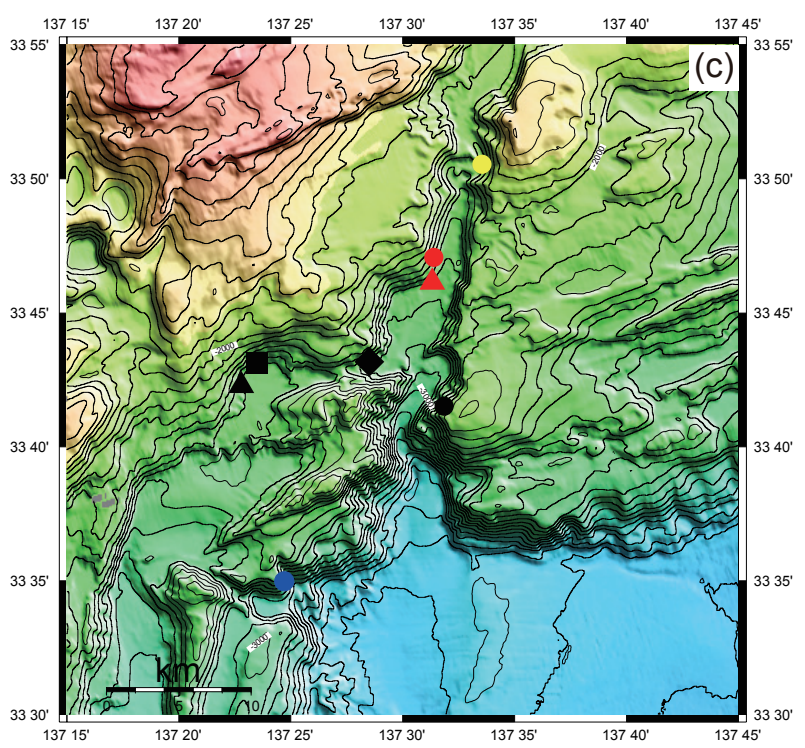

Fig. 1. (a) Schematic map showing the plate motion around Japan Islands. A square indicates the range of Fig. 1b. (b) Bathymetric map showing the eastern Nankai Trough. A square indicates the range of Fig. 1c. (c) Close up of the Tenryu Submarine Canyon showing sampling sites during the YK05-08 cruise. Red plots indicate the locations of sites where bacterial mat were found on the seafloor. A blue one indicates the location of a site where a Calyptogena colony was found. Black ones indicate those where nothing special was found on the seafloor. A yellow one indicates where a bunch of bananas was found on the seafloor. 
Thrust, and Tenryu Frontal Thrust, are all active, and several chemosynthesis-based benthic communities have been discovered along the faults (Kawamura et al., 1998; Le Pichon et al., 1987; Sibuet et al., 1988).

\section{Samples}

For our samples, we collected MBARI-type push cores (http://www.mbari.org/push-cores/; length = $35 \mathrm{~cm}$ ) from the surface of the seafloor in the Tenryu Submarine Canyon during the YK05-08 cruise of the $R / V$ Yokosuka (JAMSTEC) between June 20, 2005 and July 08, 2005. Sampling sites are shown in Table 1 and Fig. 1c. Detailed tracks and photos have been reported in Kawamura et al. (2009). We conducted 6 dives, and sampled 8 cores during the cruise (Table 1); 2 cores from bacterial mats, 1 core from Calyptogena colony, and 4 cores from the normal seafloor for a reference. During dive 892, we found a white patch on the seafloor in the Tenryu Canyon (Fig. 2a), and sampled the surface sediment by a push core from the patch (Fig. 2b). Subsequently, we retrieved a bunch of bananas from the patch (Figs. 2c and 2d). The sediment sample with a length of $14.5 \mathrm{~cm}$ was obtained from around the patch, of which $5 \mathrm{~cm}$ of the surface above comprised black mud including a fragment of a banana, which had a strong odor like hydrogen sulfide $\left(\mathrm{H}_{2} \mathrm{~S}\right)$. As shown in Fig. 3 , below $5 \mathrm{~cm}$, we found light gray clay without any odor.

\section{Analytical methods}

We squeezed the sediment in a $60-\mathrm{cm}^{3}$ syringe using a C-clamp to extract the pore water into a $10-\mathrm{cm}^{3}$ syringe as quickly after recovery as possible (Manheim, 1968). Subsequently, the pore water was distributed into two bottles for water and gas analyses. Using aliquot for water analysis, we measured both the ammonia $\left(=\mathrm{NH}_{3}+\mathrm{NH}_{4}^{+}\right)$ concentration and $\mathrm{pH} /$ alkalinity using standard shipboard techniques and procedures (Gamo and Gieskes, 1992; Gieskes et al., 1991). When these samples were brought back to our onshore laboratory at Hokkaido University, we measured the concentrations of chloride $\left(\mathrm{Cl}^{-}\right)$and sulfate $\left(\mathrm{SO}_{4}{ }^{2-}\right)$ ions using ion chromatography (Tsunogai and Wakita, 1995). Further, the concentration and carbon isotope ratio $\left({ }^{13} \mathrm{C} /{ }^{12} \mathrm{C}\right)$ of $\mathrm{CH}_{4}$ were determined using an
irm-GC/MS system (HP6890 and Finnigan MAT252) with an open-split interface (Finnigan Combustion III with some modifications) (Tsunogai et al., 2002). Next, we calculated the concentration of $\Sigma \mathrm{CO}_{2}\left(=\mathrm{H}_{2} \mathrm{CO}_{3}+\mathrm{HCO}_{3}{ }^{-}+\mathrm{CO}_{3}{ }^{2-}\right)$ using these $\mathrm{pH}$ and alkalinity values. Finally, we determined the ${ }^{13} \mathrm{C} /{ }^{12} \mathrm{C}$ value of $\Sigma \mathrm{CO}_{2}$ using the irm-GC/MS system (Ijiri et al., 2003). The ${ }^{13} \mathrm{C} /{ }^{12} \mathrm{C}$ value was calibrated to the Vienna Pee Dee Belemnite (VPDB) standard and converted into conventional delta notation $\left(\delta^{13} \mathrm{C}\right)$ using the standard correction procedure given below (Coplen, 2011):

$$
\delta^{13} \mathrm{C}=\left(\left({ }^{13} \mathrm{C} /{ }^{12} \mathrm{C}\right)_{\text {sample }} /\left({ }^{13} \mathrm{C} /{ }^{12} \mathrm{C}\right)_{\mathrm{VPDB}}\right)-1
$$

The analytical precisions are shown in Table 1.

\section{Results}

From our analyses, we found that $\mathrm{Cl}^{-}$ concentrations showed a value that is equivalent to that of overlying seawater (Fig. 4a). Further, $\mathrm{SO}_{4}{ }^{2-}$ remarkably declined to zero at $15 \mathrm{~cm}$ below the seafloor ( $\mathrm{cmbsf})$ at the bacterial mat site, and sulfate was a little lower than that of overlying seawater at $1.5 \mathrm{cmbsf}$ at the banana site; however, at the other sites, $\mathrm{SO}_{4}{ }^{2-}$ showed values equivalent to that of overlying seawater (Fig. 4b). Further, as shown in Fig. 4d, the concentrations of $\Sigma \mathrm{CO}_{2}$ were high at the surface of the banana site and the deep layer of the bacterial mat site. Similarly, $\mathrm{CH}_{4}$ concentrations were high at the bacterial mat site and the Calyptogena site (Fig. $4 \mathrm{e}$ ). The $\delta^{13} \mathrm{C}$ values of $\mathrm{\Sigma CO}_{2}\left(\delta^{13} \mathrm{C}_{\mathrm{CO} 2}\right)$ decreased to $-50 \%$ at $15 \mathrm{cmbsf}$ at the bacterial mat site and again increased below $15 \mathrm{cmbsf}$ (Fig. 4f). In addition, the $\delta^{13} \mathrm{C}_{\mathrm{CO} 2}$ values decreased to $-20 \%$ o at the Calyptogena site and decreased to approximately $-15 \%$ at the surface of the banana site. Finally, the $\delta^{13} \mathrm{C}_{\mathrm{CH} 4}$ values declined to $-80 \%$ at the bacterial mat; however, they nearly stayed constant between $-60 \%$ and $-40 \%$ at the other sites (Fig. 4g). Ammonia increased gradually with depth in all cores (Fig. 4c).

\section{Discussion}

In Fig. 5, we plotted the relation between ammonia and $\Sigma \mathrm{CO}_{2}$ concentrations in the pore water for each site in this study. When organic matter derived from marine 

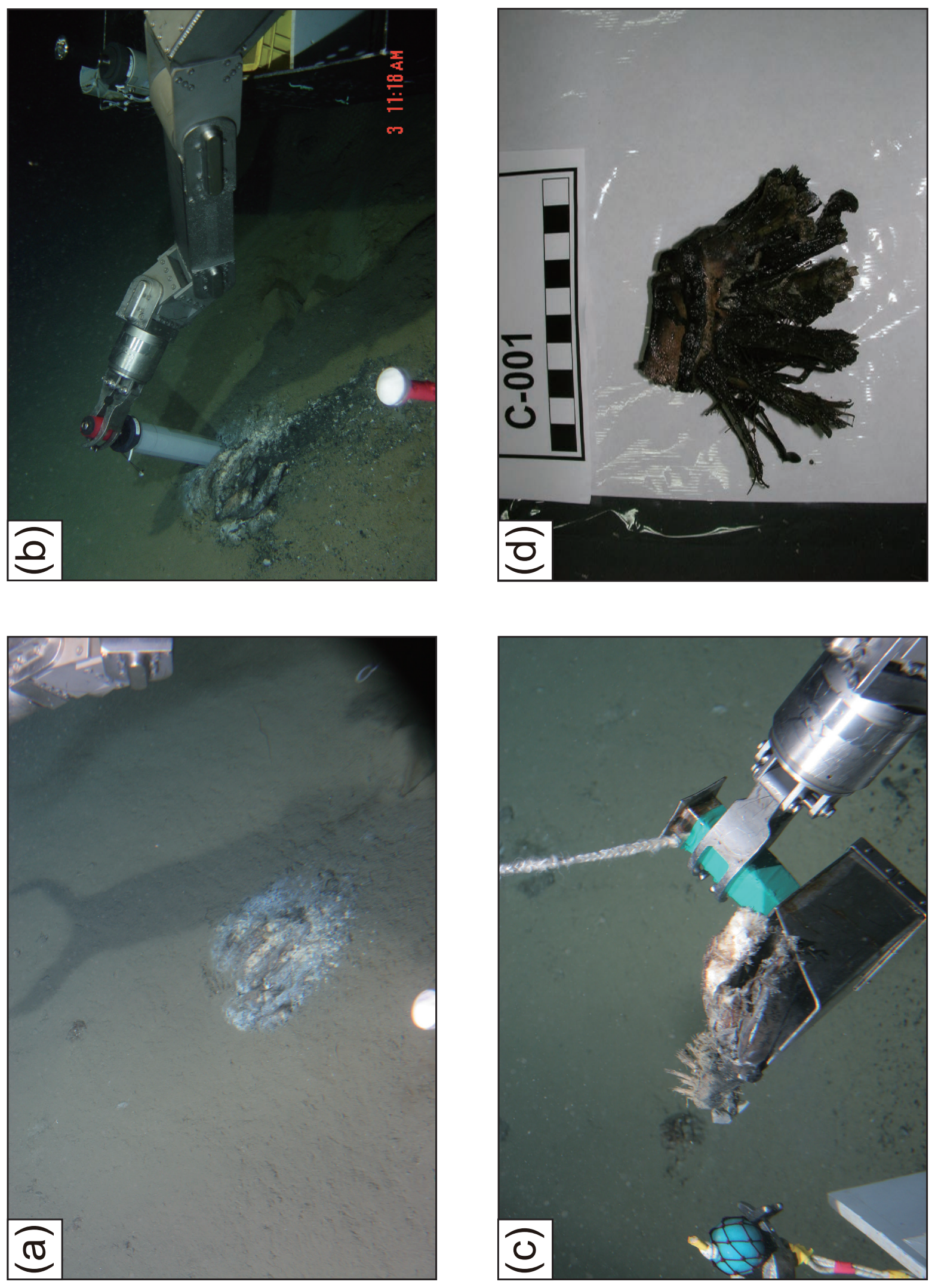

\&

$\Xi$

콩

용

‡ี :

등

용

를

苛

寻

进

롱

$3 \frac{0}{3}$

ᄒ ․ํ

0

के व

क्षे :

类

$\pm$.

Е

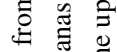

ฮ స్ㅠㅀ

氜

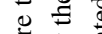

昰 品

« 言 윰

ङ

의

i 
organisms is decomposed in the sediment, it reacted according to the equation given below, generating $\Sigma \mathrm{CO}_{2}$ and ammonia at a ratio of $106: 16$, i.e., the Redfield ratio (Redfield, 1934):

$$
\begin{aligned}
2\left(\mathrm{CH}_{2} \mathrm{O}\right)_{106}\left(\mathrm{NH}_{3}\right)_{16} & +106 \mathrm{SO}_{4}{ }^{2-} \\
= & 212 \mathrm{HCO}_{3}{ }^{-}+106 \mathrm{H}_{2} \mathrm{~S}+32 \mathrm{NH}_{3}
\end{aligned}
$$

The Redfield ratio was based on organic matter in seawater (Redfield, 1934), but in interstitial water, ammonia adsorbs to a surface of sediment particles (Rosenfeld, 1979), or a ratio of carbon to nitrogen $(\mathrm{C} / \mathrm{N}$ ratio) of sediment shows regionally variation (e.g., Sampei and Matsumoto, 2001). Considering these circumstances, we showed the normal data as a reference by small crosses in Fig. 5. These data are unpublished data obtained during YK02-02, YK03-03,
YK05-08, YK06-03, and YK10-09 cruises. The analytical methods and the sampling procedures are the same as those of this study, and the sampling points have already been reported (Toki et al., 2014; Toki et al., 2004). The "normal data" mean chemical compositions of interstitial water from sediments of the seafloor without bacterial mats, tube worms, Calyptogena colony, or carbonate chimney.

Considering that the $\Sigma \mathrm{CO}_{2}$ concentration in overlying seawater was approximately $2.4 \mathrm{mmol} / \mathrm{L}$ and ammonia was less than $100 \mu \mathrm{mol} / \mathrm{L}$ (Table 1), we draw a regression line through those of seawater as a dashed line in Fig. 5. The slope is 6.3 , which is slightly smaller than the Redfield ratio $(106 / 16=6.625)$, but the deviation of $4.5 \%$ is corresponding to anything more than the analytical errors (Table 1). Hereafter, we recognize the regression line as the standard, and discuss the ratio of $\Sigma \mathrm{CO}_{2}$ concentration to ammonia concentration in the other sites. The ratios in
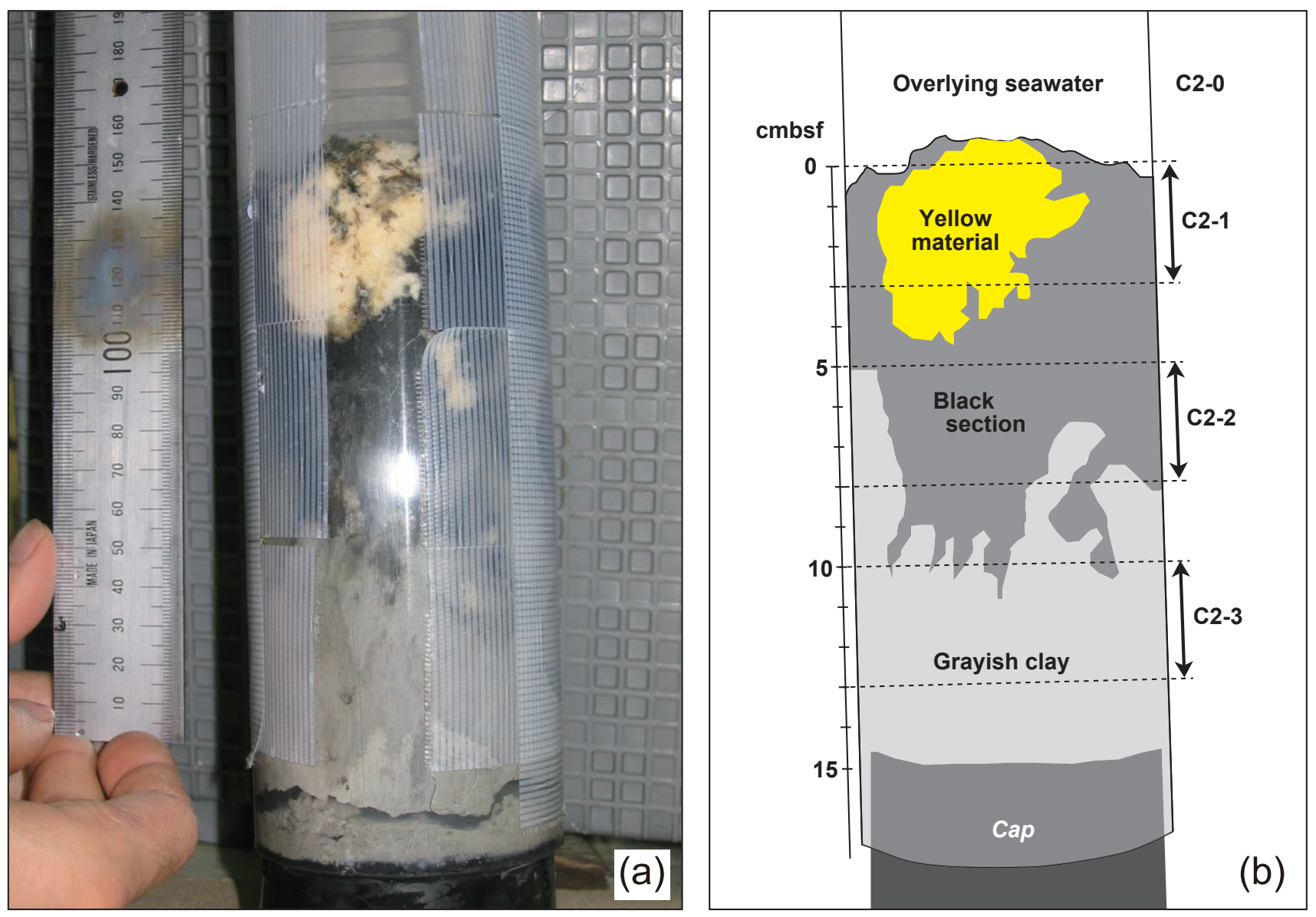

Fig. 3. (a) A photo from a lateral view of sediment sample cored from the deep-sea floor around the bananas in the Tenryu Canyon. (b) A description about the cored sediment shown in Fig. 3a; there is yellow material above $\sim 4.5 \mathrm{~cm}$ from the top, followed by a black section above $\sim 10.5 \mathrm{~cm}$ which is probably containing iron sulfides (Gieskes et al., 2005; Gieskes et al., 2011), below which the sediment becomes grayish in color (normal sediment). Total length is $\sim 14.5 \mathrm{~cm}$. Dashed lines indicate the sample intervals; $0-3 \mathrm{~cm}$ for C2-1 sample, $5-8 \mathrm{~cm}$ for C2-2 sample, and 10-13 cm for C2-3 sample, as listed in Table 1. The depths of the samples in Table 1 correspond to averages of the intervals of the samples. 

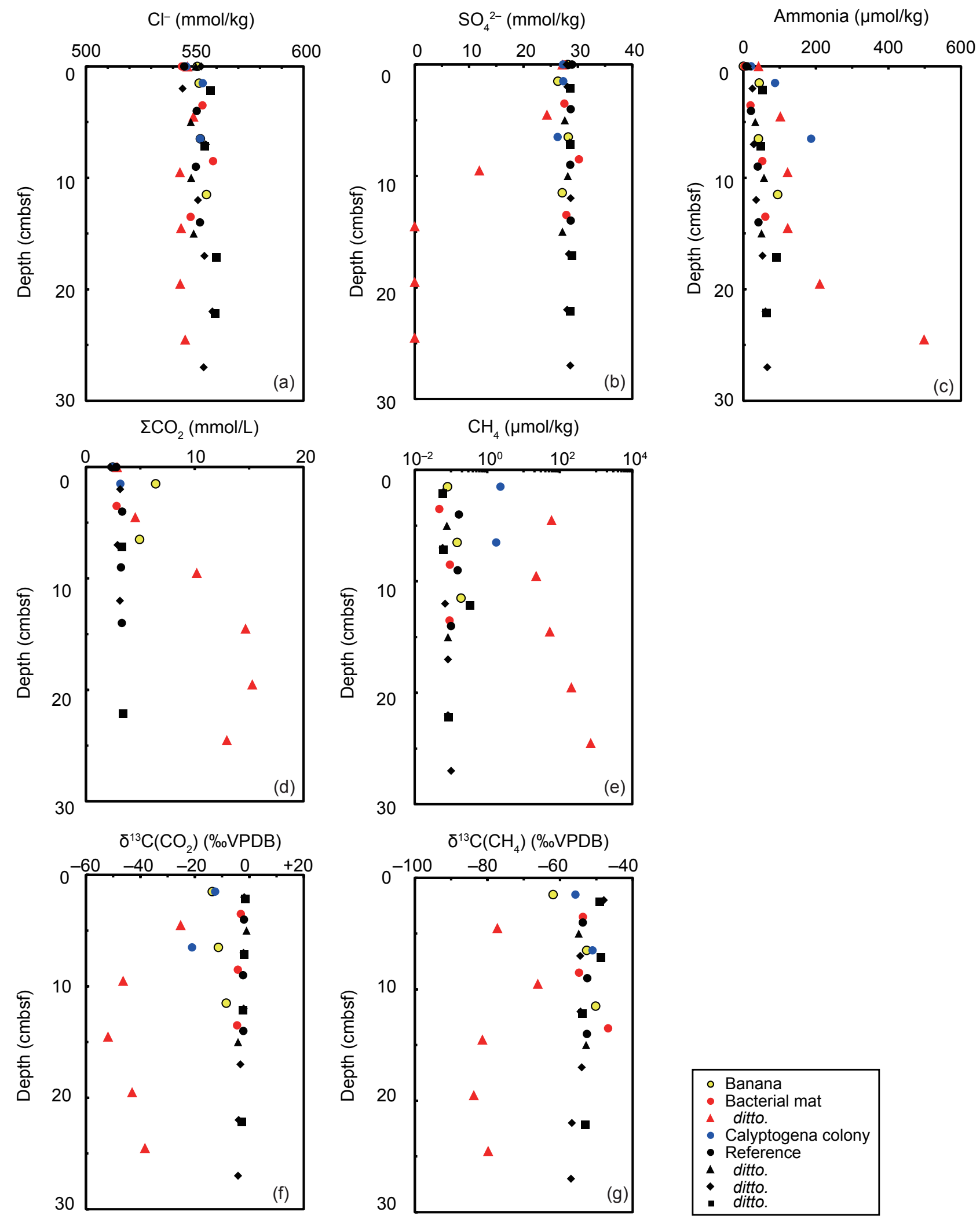

- Banana

- Bacterial mat

- ditto.

- Calyptogena colony

- Reference

- ditto.

ditto.

- ditto.

Fig. 4. Vertical profiles of chemical and isotopic compositions of the pore water. A yellow plot represents the data of chemical compositions in the interstitial water from the banana site; red ones from the bacterial mat sites, a blue one from the Calyptogena site, and black ones from the normal seafloor. 


\begin{tabular}{|c|c|c|c|c|c|c|c|c|}
\hline 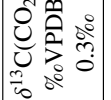 & I & $1 \bar{i}, \bar{F}$ & | $\vec{i} \bar{i} \vec{i} \hat{i} \vec{i} \vec{i}$ & $1, \vec{i} \vec{i}, \hat{i}$ & $1 \stackrel{0}{\substack{0 \\
1}}$ & | $\bar{i} \stackrel{9}{i}+\frac{n}{1}$ & 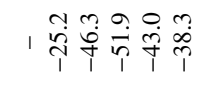 & 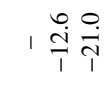 \\
\hline 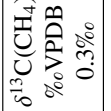 & 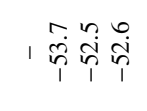 & , 1 站 | & 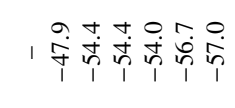 & 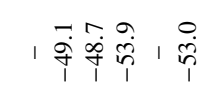 & 1 & I & 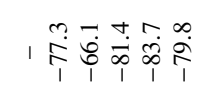 & , $\stackrel{\infty}{i n}_{i}=$ \\
\hline 苟高 & क्षल ले ले & 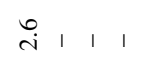 & 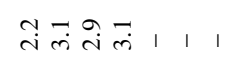 & $11 \stackrel{m}{m} 11 \stackrel{+}{m}$ & 访苛字 & $\stackrel{\infty}{i} \underset{i}{\infty} \quad|\quad|$ & 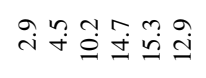 & तुल \\
\hline 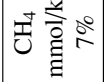 & $1 \frac{0}{0} \div \frac{1}{0} \div 0$ & $1 \stackrel{\infty}{\circ}, \stackrel{\infty}{0}$ & । & । & $1 \stackrel{\infty}{0}: \frac{n}{0} \div$ & 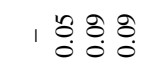 & | निं तुं & 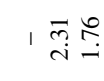 \\
\hline 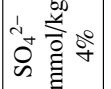 & के & 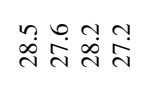 & 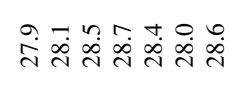 & 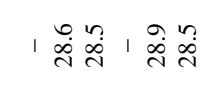 & 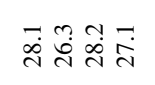 & 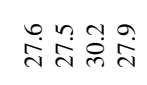 & 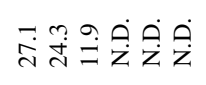 & ma \\
\hline 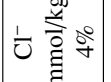 & 告话品会 & 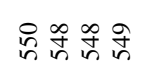 & 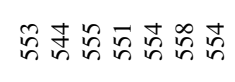 & 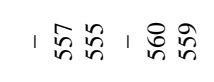 & 吾垥会合 & 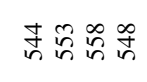 & 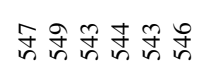 & 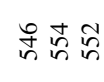 \\
\hline 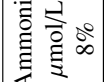 & क्षें & 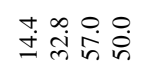 & 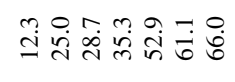 & 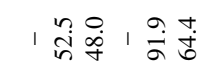 & 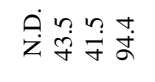 & 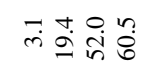 & 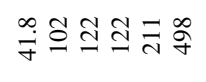 & 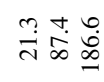 \\
\hline 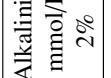 & 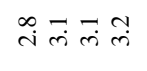 & 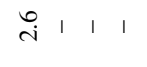 & 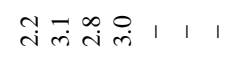 & $11 \bar{m} 11 \%$ & 说字 1 & $\hat{i} \hat{i} \mid 1$ & 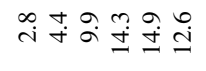 & $\stackrel{n}{i} \bar{m} 1$ \\
\hline $\begin{array}{ll}\text { 돌 } & 0 \\
0\end{array}$ & 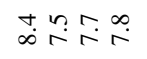 & $\begin{array}{lllll}2 & 1 & 1 & 1\end{array}$ & 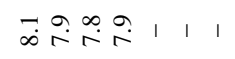 & 1 1 & 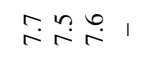 & $\bar{\infty}=1$ & ஸિ & 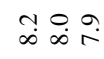 \\
\hline 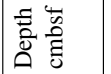 & 。요용 & o & o irir & | & 은 & 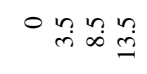 & 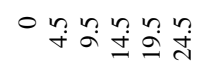 & 0 은 \\
\hline$\dot{z}$ & $O-N m$ & $O-N m$ & $0-N m+n 0$ & $0-\alpha m+n$ & $0-\mathrm{Nm}$ & $0-N m$ & $0-N m+n$ & $0-4$ \\
\hline 言 & $\bar{U}$ & $\bar{U}$ & $\tilde{0}$ & $\tilde{U}$ & $\tilde{U}$ & $\tilde{O}$ & $\tilde{3}$ & $\tilde{3}$ \\
\hline 总 & 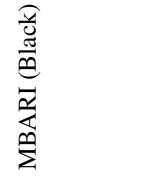 & 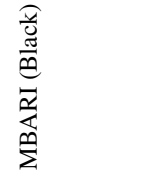 & 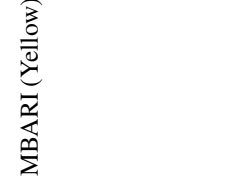 & 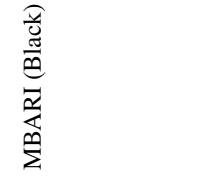 & 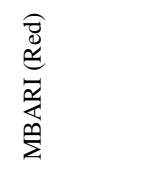 & 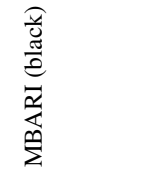 & 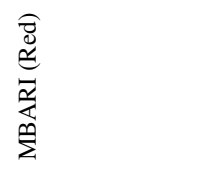 & 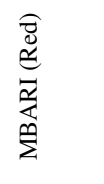 \\
\hline 袬 & ฟָ̃ & $\overline{\tilde{n}}$ & 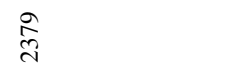 & 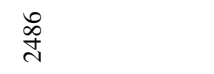 & $\stackrel{4}{\frac{\pi}{4}}$ & $\underset{n}{\infty}$ & 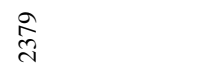 & 离 \\
\hline 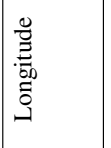 & $\begin{array}{l}\stackrel{+}{0} \\
\infty \\
\dot{m} \\
\stackrel{\infty}{\cong}\end{array}$ & $\begin{array}{l}\text { Dे } \\
0 \\
0 \\
\text { d } \\
\hat{m} \\
\underline{-}\end{array}$ & 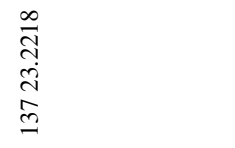 & 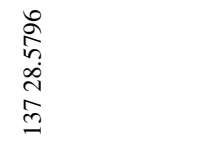 & $\begin{array}{l}\hat{\delta} \\
\text { ch } \\
\stackrel{m}{0} \\
\hat{n}\end{array}$ & $\begin{array}{l}\stackrel{\infty}{ } \\
\stackrel{\infty}{+} \\
\stackrel{+}{m} \\
\stackrel{\sim}{m}\end{array}$ & $\begin{array}{l}\stackrel{0}{0} \\
m \\
m \\
m \\
m\end{array}$ & $\begin{array}{l}\text { वे } \\
\text { d. } \\
\stackrel{d}{1} \\
\stackrel{0}{a}\end{array}$ \\
\hline & 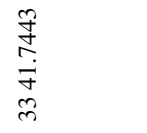 & 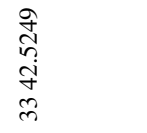 & 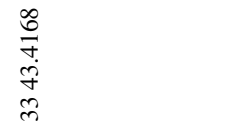 & 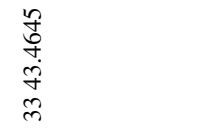 & 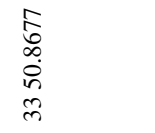 & $\begin{array}{l}\bar{\Sigma} \\
i \\
i j \\
b \\
\tilde{m}\end{array}$ & $\begin{array}{l}\frac{\infty}{2} \\
\stackrel{\vec{j}}{+} \\
\dot{j} \\
\end{array}$ & $\begin{array}{l}\stackrel{m}{m} \\
\stackrel{0}{0} \\
\infty \\
m \\
m\end{array}$ \\
\hline 总 & 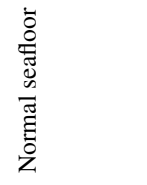 & 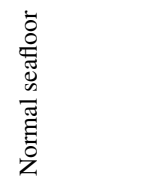 & 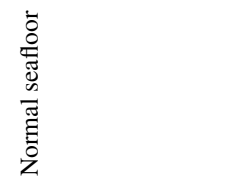 & 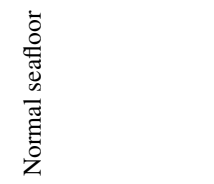 & 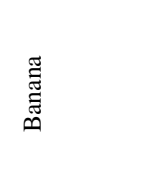 & 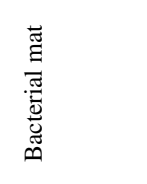 & 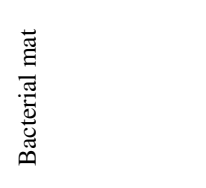 & 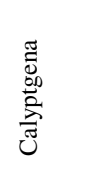 \\
\hline 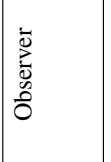 & 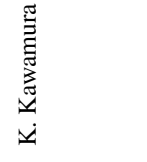 & 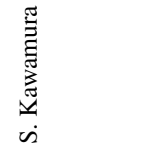 & & 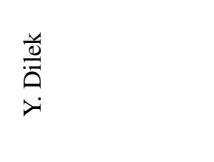 & 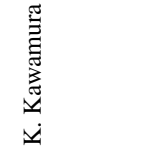 & 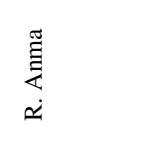 & & 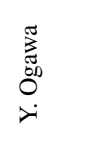 \\
\hline$\stackrel{D}{\geq}$ & $\begin{array}{l}\infty \\
\infty \\
\infty\end{array}$ & $\underset{\substack{\infty \\
\infty}}{\infty}$ & & $\stackrel{\infty}{\infty}$ & ڤิ & $\hat{\infty}$ & & S̊ \\
\hline ڤัّ & 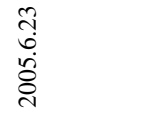 & 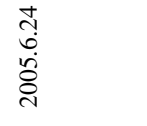 & & 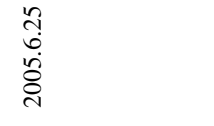 & $\begin{array}{l}\text { ?. } \\
\stackrel{2}{8} \\
\stackrel{i}{8}\end{array}$ & $\begin{array}{l}n \\
\stackrel{n}{0} \\
\stackrel{8}{8}\end{array}$ & & 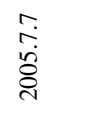 \\
\hline
\end{tabular}


the pore water from the Calyptogena and reference sites in this study are comparable to those of the standard ratio. However, at the banana and bacterial mat sites, a surplus $\Sigma \mathrm{CO}_{2}$ is distributed to an extent beyond the degradation of organic materials by marine organisms. This surplus was also detected at cold seep areas (Kulm et al., 1986) due to anaerobic methane oxidation (AOM) from $\Sigma \mathrm{CO}_{2}$ without ammonia generation, which is governed by the following equation:

$$
\mathrm{CH}_{4}+\mathrm{SO}_{4}{ }^{2-}=\mathrm{HCO}_{3}{ }^{-}+\mathrm{HS}^{-}+\mathrm{H}_{2} \mathrm{O}
$$

At the bacterial mat site, the $\mathrm{CH}_{4}$ concentration was considerably high and the $\mathrm{SO}_{4}{ }^{2-}$ concentration significantly diminished (Figs. $4 \mathrm{~d}$ and $4 \mathrm{~b}$ ), suggesting that high $\Sigma \mathrm{CO}_{2}$ concentration detected at the bacterial mat site resulted from AOM. In addition, when AOM occurred, $\delta^{13} \mathrm{C}_{\mathrm{CO} 2}$ values as low as $-30 \%$ were detected in the pore water (Martin et al., 1997; Suess and Whiticar, 1989). At the bacterial mat site, the $\delta^{13} \mathrm{C}_{\mathrm{CO} 2}$ values showed considerably low values (less than $-50 \%$ ), as shown in Fig. 4f; however, at the banana site, such features were not observed. Instead, the $\Sigma \mathrm{CO}_{2}$ concentration was higher (Fig. $4 \mathrm{~d}$ ) and the $\delta^{13} \mathrm{C}_{\mathrm{CO} 2}$ value was lower (Fig. 4f) than those of the reference sites, respectively, without a high-level $\mathrm{CH}_{4}$ concentration. This suggests that high $\Sigma \mathrm{CO}_{2}$ concentration with a relatively low

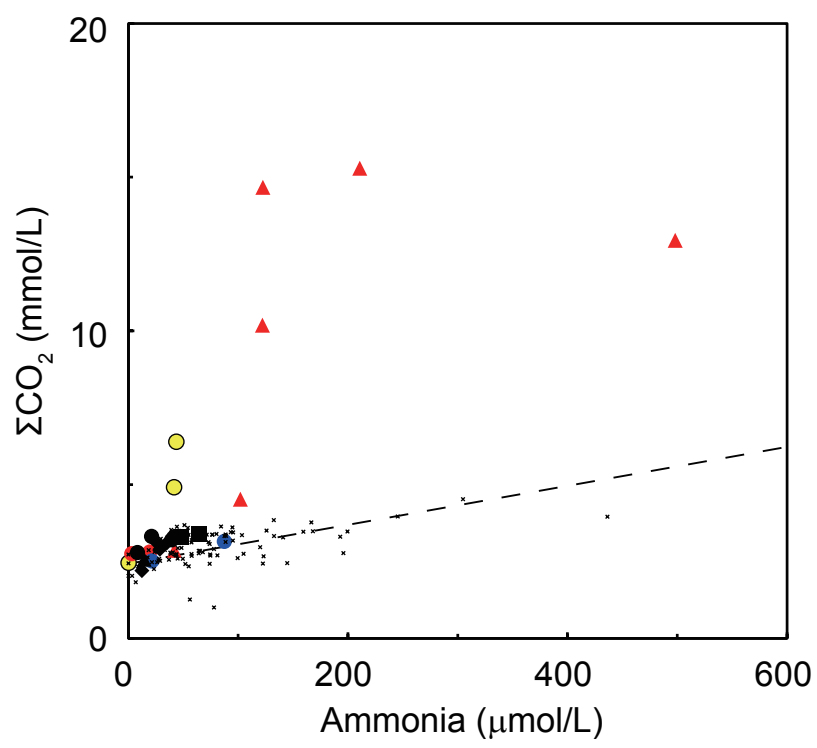

Fig. 5. Relation between ammonia and $\Sigma \mathrm{CO}_{2}$ concentrations in the pore water. Symbols as in Fig. 4, together with the data from the normal seafloor in the Nankai Trough as small crosses (see the main text for more information). $\delta^{13} \mathrm{C}_{\mathrm{CO} 2}$ value was not due to the presence of $\mathrm{CH}_{4}$, which needs a carbon source other than $\mathrm{CH}_{4}$ at the banana site.

Given the above, we first argue where the carbon source is in the sediment column at the banana site. When we investigate the vertical profiles shown in Fig. 4, we can find a peak of $\Sigma \mathrm{CO}_{2}$ concentration at the top of the sediment column with a relatively low $\delta^{13} \mathrm{C}$ value, as shown in Figs. $4 \mathrm{~d}$ and $4 \mathrm{f}$, indicating that the carbon source at the banana site is located at the top of the column. In general, terrestrial plants have high $\mathrm{C} / \mathrm{N}$ ratios and low $\delta^{13} \mathrm{C}$ values relative to those of marine organisms (Fry and Sherr, 1984; Hedges et al., 1986; Parsons et al., 1961). Further, banana is one of the terrestrial plants with a $\mathrm{C} 3$ cycle and has a $\mathrm{C} / \mathrm{N}$ ratio of approximately 30 and a $\delta^{13} \mathrm{C}$ value of approximately $-30 \%$ o (Ilori et al., 2007; Tixier et al., 2013). Based on these facts, the degradation of the banana on the seafloor would cause surplus $\Sigma \mathrm{CO}_{2}$ generation at the surface sediment of the banana site. In particular, the surface sediment after its recovery smelled like $\mathrm{H}_{2} \mathrm{~S}$, suggesting that sulfate reduction occurred for generating $\mathrm{H}_{2} \mathrm{~S}$ at the surface sediment, which is expressed in the following equation:

$2\left(\mathrm{CH}_{2} \mathrm{O}\right)_{30}\left(\mathrm{NH}_{3}\right)+30 \mathrm{SO}_{4}{ }^{2-}=60 \mathrm{HCO}_{3}{ }^{-}+30 \mathrm{H}_{2} \mathrm{~S}+2 \mathrm{NH}_{3}$,

where a banana represents $\left(\mathrm{CH}_{2} \mathrm{O}\right)_{30}\left(\mathrm{NH}_{3}\right)$, because it is one of the terrestrial plants having a $\mathrm{C} / \mathrm{N}$ ratio of $\sim 30$ (Ilori et al., 2007). In addition, the generated $\mathrm{H}_{2} \mathrm{~S}$ could react with $\mathrm{Fe}$ ions to form a precipitate by the following stoichiometric formula (Gieskes et al., 2005; Gieskes et al., 2011):

$$
1 / 3 \mathrm{Fe}_{2} \mathrm{O}_{3}+\mathrm{H}^{+}+\mathrm{HS}^{-}=2 / 3 \mathrm{FeS}+1 / 3 \mathrm{~S}^{0}+\mathrm{H}_{2} \mathrm{O} .
$$

The produced iron sulfide could be distributed as a black mud at $0-10.5 \mathrm{cmbsf}$ (Fig. 3). Native sulfur is theoretically produced as a by-product, but we could not find it in the sampled sediments (Fig. 3). Indeed sulfate concentration slightly decreased at $1.5 \mathrm{~cm}(+/-1.5 \mathrm{~cm})$ below the seafloor (Fig. 4b), but the black mud stretched to deeper as shown in Fig. 3, which could be due to artificial "bioturbation". The black mud was very fine and buoyant, while the underlying clay was relatively hard. As a coring had been conducted, the black mud possibly was dragged along the inner of the corer, so the black mud could seem to stretch to deeper. Thus, sulfate reduction would occur near the deeper part of the bananas (eq. 4), however, considering the bananas were placed on the seafloor (Fig. 2), the upper part of the bananas 
would undergo decomposition by dissolved oxygen, aerobic oxidation. This is expressed in the following equation:

$$
\left(\mathrm{CH}_{2} \mathrm{O}\right)_{30}\left(\mathrm{NH}_{3}\right)+30 \mathrm{O}_{2}=30 \mathrm{CO}_{2}+30 \mathrm{H}_{2} \mathrm{O}+\mathrm{NH}_{3} \text {. }
$$

The ratio of $\Sigma \mathrm{CO}_{2}$ to ammonia generated by the aerobic oxidation according to Eq. (6) is also stoichiometrically 30, explaining the excess $\Sigma \mathrm{CO}_{2}$ relative to the marine organism degradation observed at the banana site.

Based on the above considerations, we inferred the redox reaction around the bananas (Fig. 6), where aerobic and anaerobic oxidation occurs in a locally organic-rich environment. This environment could be caused by the bananas sinking down to the deep-sea floor. Since bananas are a tropical plant and they don't grow wild around this area in Japan, possible route of the bananas could be (1) dropping down from a tree and carried on the Kuroshio Current, or (2) dropping from a cargo ship during shipping. The Kuroshio Current is one of the strongest current in the world flowing through the west side of the North Pacific Ocean, and it flows from the east coast of Philippines to northeast off Japan. In the former case, bananas dropped down from a tree could have been flowed on the current, and have sunk down at the site. The Kuroshio Current snakes the way day by day, and the pathway is reported every day on a website of Japan Meteorological Agency. Data of an axis of the Kuroshio can be seen on another website (http://www.mirc.jha.or.jp/), and the Kuroshio axis draft away from the Japan coast, and it flowed through far south from the banana site during the sampling campaign in

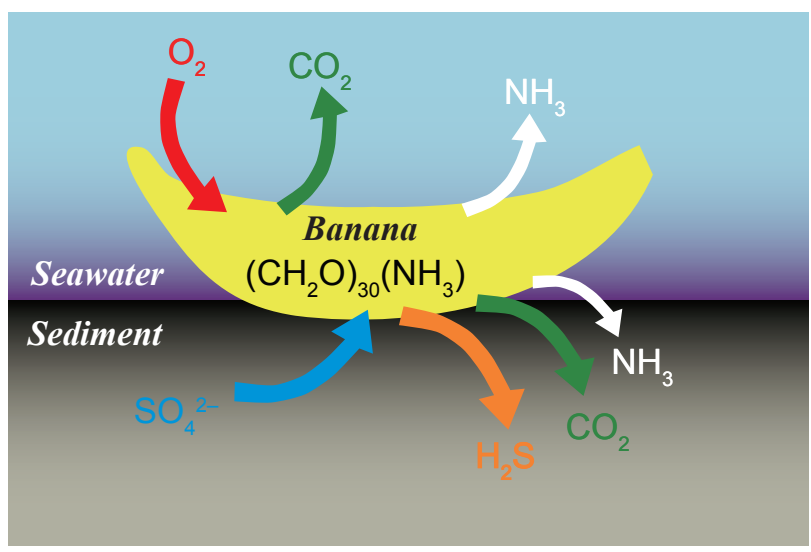

Fig. 6. Schematic showing microbial metabolism around the bananas in the Tenryu Canyon.
July 2005. The Kuroshio snake has initiated since August 2004. Therefore, if the bananas had been carried on the Kuroshio axis, they could have been dropped earlier than July 2004. But if the bananas were attributable to such a natural event, much more bananas should be widespread along the Kuroshio Current. We have never heard of dropped bananas on the deep-sea floor, and the phenomenon could be caused from an accident, that is, the second possible route. We can monitor the positions of sailing ships on a website (http://www.marinetraffic.com/), and some ships are sailing over the banana site based on the data. Probably, the bananas could be dropped down from one of these ships. To this point, this phenomenon was responsible for having an anthropogenic impact on the deep-sea floor ecosystem. For deep-sea floor bacteria intrinsically earning a sparse living by utilizing considerably little organic matter, the bananas could have been a "treat from heaven" and the bacteria could gather together around the bananas from the ambient seawater or pore water. The bacterial mat, however, could not flourish on a sustainable basis because the bananas had finite mass. The bacteria clustering around the bananas could be back to the seawater or pore water and live on little organic matter. Such an incidental phenomenon could not have a significant impact on the deep-sea floor ecosystem because the phenomenon has a low frequency of occurrence. Conversely, if this phenomenon had a high frequency of occurrence, it would create substantial contact between anthropogenic materials and deep-sea floor ecosystems; thus, anthropogenic materials having a substantial impact on the deep-sea floor ecosystem cannot be denied. We must continue to observe this impact without missing uncommon phenomenon to understand the influence of this phenomenon on deep-sea environments during seafloor exploration.

\section{Conclusion}

During the YK05-08 cruise, we collected a surface sediment around a bunch of bananas discovered in the Tenryu Canyon. The sediment was squeezed to extract the pore water just after recovery. Then, we measured ammonia, $\mathrm{pH} /$ alkalinity, $\mathrm{Cl}^{-}, \mathrm{SO}_{4}{ }^{2-}, \mathrm{CH}_{4}$ concentrations, and $\delta^{13} \mathrm{C}$ of $\mathrm{CH}_{4}$ and $\Sigma \mathrm{CO}_{2}$ in the pore water. Based on the vertical profiles of chemical components in the pore water, $\mathrm{CH}_{4}$ and $\Sigma \mathrm{CO}_{2}$ increased, whereas $\mathrm{SO}_{4}{ }^{2-}$ decreased in the deeper part of the sediment column at the bacterial mat site. In contrast, 
considerably high $\Sigma \mathrm{CO}_{2}$ concentration was detected at the surface of the column at the banana site. Based on the ratios of $\Sigma \mathrm{CO}_{2}$ to ammonia, an excess of $\Sigma \mathrm{CO}_{2}$ was detected that was unable to be explained by organic matter degradation from marine organisms at the banana and bacterial mat sites.

Further, as reported in cold seep areas, AOM could result in excess $\Sigma \mathrm{CO}_{2}$ at the bacterial mat, where $\mathrm{CH}_{4}$ was enriched, $\mathrm{SO}_{4}{ }^{2-}$ diminished, and $\delta^{13} \mathrm{C}_{\mathrm{CO} 2}$ was lower than $-50 \%$ in the deeper side of the column; these results support the AOM occurrence at the bacterial mat site. On the other hand, at the banana site, $\mathrm{CH}_{4}$ was at a normal level for deep-sea environments, implying the input of a carbon source rather than methane oxidation. This carbon source would be located at the top of the sediment column based on the vertical profiles of chemical constituents in the pore water at the banana site. Bananas, one of the terrestrial plants, have higher $\mathrm{C} / \mathrm{N}$ ratios than those of marine organic matter; thus bananas could be a possible carbon source during degradation. At the banana site, the bananas broke down to form $\Sigma \mathrm{CO}_{2}$, thereby leading to a high-concentration anomaly of $\Sigma \mathrm{CO}_{2}$ at the surface of the banana site. Despite the strong $\mathrm{H}_{2} \mathrm{~S}$ odor and black sulfide precipitation at the surface sediment of the banana site, sulfate depletion was a little observed in the pore water, suggesting that the bananas broke down using sulfate and oxygen in the deep-sea water and the interstitial water in the sediment. Therefore, the bananas would extraordinarily enrich the organic matter in the deep-sea environment, building a highly condensed microbial community on the deep-sea floor.

\section{Acknowledgements}

This study was partially supported by the Grantin-Aid for Scientific Research A (No. 16204045) and the Exploratory Research (No. 15K13603). We wish to express my sincere thanks to Professor J. Gieskes and Dr. Ijiri for a careful review and constructive comments. We are particularly grateful to M. Tsutsumi (ORI) and T. Noguchi (Kyoto University), K. Okamura (Kochi University), and Y. Nishio (KCC) for their generous support, stimulating discussions, and valuable suggestions throughout our study. We also gratefully acknowledge Y. Ogawa and R. Anma (Tsukuba University) and A. Yamaguchi (University of Tokyo) for their help in collecting samples. Further, we thank the captain and crew of $\mathrm{R} / \mathrm{V}$ Yokosuka, and the commander and operation team of the submersible Shinkai6500 during the YK05-08 cruise for their continued dedication to marine geochemical work. The authors would like to thank Enago (www.enago.jp) for the English language review. Finally, we express our sincere thanks to Dr. Oguri, one of the JAMSTEC-R editorial board, for encouraging writing and productive suggestions.

\section{References}

Coplen, T.B. (2011), Guidelines and recommended terms for expression of stable-isotope-ratio and gas-ratio measurement results, Rapid Communications in Mass Spectrometry, 25, 2538-2560.

Dando, P.R., A.J. Southward, E.C. Southward, D.R. Dixon, A. Crawford, and M. Crawford (1992), Shipwrecked tube worms, Nature, 356, 667.

Fry, B. and B.F. Sherr (1984), $\delta^{13} \mathrm{C}$ measurements as indicators of carbon flow in marine and freshwater ecosystems, Contributions in marine science, 27, 13-47.

Gage, J.D. and P.A. Tyler (1991), Deep sea biology: a natural history of organisms at the deep-sea floor, Cambridge University Press, 504p.

Gamo, T. and J.M. Gieskes (1992), Shipboard chemical analyses of sedimentary pore waters during the Ocean Drilling Program (ODP) Leg 131, Chikyukagaku (Geochemistry), 26, 1-15.

Gieskes, J., C. Mahn, S. Day, J.B. Martin, J. Greinert, T. Rathburn, and B. McAdoo (2005), A study of the chemistry of pore fluids and authigenic carbonates in methane seep environments: Kodiak Trench, Hydrate Ridge, Monterey Bay, and Eel River Basin, Chemical Geology, 220, 329-345.

Gieskes, J., A.E. Rathburn, J.B. Martin, M.E. Pérez, C. Mahn, J.M. Bernhard, and S. Day (2011), Cold seeps in Monterey Bay, California: Geochemistry of pore waters and relationship to benthic foraminiferal calcite, Applied Geochemistry, 26, 738-746.

Gieskes, J.M., T. Gamo, and H. Brumsack (1991), Chemical methods for interstitial water analysis aboard JOIDES Resolution, Ocean Drilling Program Texas A\&M University Technical Note, 15, 1-60.

Hedges, J.I., W.A. Clark, P.D. Quay, J.E. Richey, A.H. Devol, and M. Santos (1986), Compositions and fluxes of particulate organic material in the Amazon River, Limnology and Oceanography, 31, 717-738.

Ijiri, A., U. Tsunogai, and T. Gamo (2003), A simple method for oxygen-18 determination of milligram quantities of water 
using $\mathrm{NaHCO}_{3}$ reagent, Rapid Communications in Mass Spectrometry, 17, 1472-1478.

Ilori, M.O., S.A. Adebusoye, A.K. Iawal, and O.A. Awotiwon (2007), Production of biogas from banana and plantain peels, Advances in Environmental Biology, 1, 33-38.

Kawamura, K., Y. Ogawa, R. Anma, S. Yokoyama, S. Kawakami, Y. Dilek, G.F. Moore, S. Hirano, A. Yamaguchi, T. Sasaki, and YK05-08 Leg 2 and YK06-02 Shipboard Scientific Parties (2009), Structural architecture and active deformation of the Nankai Accretionary Prism, Japan: Submersible survey results from the Tenryu Submarine Canyon, Geological Society of America Bulletin, 121, 1629-1646.

Kawamura, K., Y. Ogawa, K. Fujikura, M. Hattori, H. Machiyama, T. Yamamoto, M. Iwai, and T. Hirono (1998), What did the "KAIKO" watch? -Detal topography and geologic structures at the mouth of Tenryu canyon-, JAMSTEC Journal of Deep Sea Research, 14, 379-388 (in Japanese with English abstract).

Kulm, L.D., E. Suess, J.C. Moore, B. Carson, B.T. Lewis, S.D. Ritger, D.C. Kadko, T.M. Thornburg, R.W. Embley, W.D. Rugh, G.J. Massoth, M.G. Langseth, G.R. Cochrane, and R.L. Scamman (1986), Oregon subduction zone: venting, fauna, and carbonates, Science, 231, 561-566.

Le Pichon, X., T. Iiyama, J. Boulègue, J. Charvet, M. Faure, K. Kano, S. Lallemant, H. Okada, C. Rangin, A. Taira, T. Urabe, and S. Uyeda (1987), Nankai Trough and Zenisu Ridge: a deep-sea submersible survey, Earth and Planetary Science Letters, 83, 285-299.

Lonsdale, P. (1977), Clustering of suspension-feeding macrobenthos near abyssal hydrothermal vents at oceanic spreading centers, Deep Sea Research, 24, $857-863$.

Manheim, F.T. (1968), Disposable syringe techniques for obtaining small quantities of pore water from unconsolidated sediments, Journal of Sedimentary Petrology, 38, 666-668.

Martens, C.S. and R.A. Berner (1974), Methane production in the interstitial waters of sulfate-depleted marine sediments, Science, 185, 1167-1169.

Martin, J.B., D.L. Orange, T.D. Lorenson, and K.A. Kvenvolden (1997), Chemical and isotopic evidence of gas-influenced flow at a transform plate boundary: Monterey Bay, California, Journal of Geophysical Research B: Solid Earth, 102, 24903-24915.

Parsons, T.R., K. Stephens, and J.D.H. Strickland (1961), On the Chemical Composition of Eleven Species of Marine Phytoplankters, Journal of the Fisheries Research Board of Canada, 18, 1001-1016.

Ranken, B., R.K. Cardwell, and D.E. Karig (1984), Kinematics of the Philippine Sea Plate, Tectonics, 3, 555-575.
Redfield, A.C. (1934), On the proportions of organic derivatives in sea water and their relation to the composition of plankton, James Johnstone Memorial Volume (Daniel, R. J. ed.), University Press of Liverpool, 176-192.

Rosenfeld, J.K. (1979), Ammonium adsorption in nearshore anoxic sediments, Limnology and Oceanography, 24, 356-364.

Sampei, Y. and E. Matsumoto (2001), C/N ratios in a sediment core from Nakaumi Lagoon, southwest Japanusefulness as an organic source indicator-, Geochemical Journal, 35, 189-205.

Seno, T. (1977), The instantaneous rotation vector of the Philippine sea plate relative to the Eurasian plate, Tectonophysics, 42, 209-226.

Sibuet, M., K.S. Juniper, and G. Pautot (1988), Cold-seep benthic communities in the Japan subduction zones: Geological control of community development, Journal of Marine Research, 46, 333-348.

Sibuet, M. and K. Olu (1998), Biogeography, biodiversity and fluid dependence of deep-sea cold-seep communities at active and passive margins, Deep Sea Research Part II: Topical Studies in Oceanography, 45, 517-567.

Smith, C.R. and A.R. Baco (2003), Ecology of whale falls at the deep-sea floor, Oceanography and Marine Biology: an Annual Review, Vol. 41 (R.N. Gibson and R.J.A. Atkinson eds.), CRC Press, 311-354.

Smith, C.R., A.G. Glover, T. Treude, N.D. Higgs, and D.J. Amon (2015), Whale-Fall Ecosystems: Recent Insights into Ecology, Paleoecology, and Evolution, Annual Review of Marine Science, Vol. 7 (Carlson, C.A. and Giovannoni, S.J. eds.), Annual Reviews, 571-596.

Suess, E., B. Carson, S.D. Ritger, J.C. Moore, M.L. Jones, L.D. Kulm, and G.R. Cochrane (1985), Biological communities at vent sites along the subduction zone off Oregon, Bulletin of the Biological Society of Washington, 6, 475-484.

Suess, E. and M.J. Whiticar (1989), Methane-derived $\mathrm{CO}_{2}$ in pore fluids expelled from the Oregon subduction zone, Palaeogeography, Palaeoclimatology, Palaeoecology, 71, 119-136.

Tixier, P., D. Dagneaux, G. Mollot, F. Vinatier, and P.F. Duyck (2013), Weeds mediate the level of intraguild predation in arthropod food webs, Journal of Applied Entomology, 137, 702-710.

Toki, T., R. Higa, A. Ijiri, U. Tsunogai, and J. Ashi (2014), Origin and transport of pore fluids in the Nankai accretionary prism inferred from chemical and isotopic compositions of pore water at cold seep sites off Kumano, Earth, Planets and Space, 66, 1-14.

Toki, T., M. Kinoshita, S. Morita, H. Masuda, H. Rashid, H. Yoshinishi, T. Nakano, and T. Noguchi (2017), The 
vertical chloride ion profile at the IODP Site C0002, Kumano Basin, off coast of Japan, Tectonophysics, 710 $711,88-96$.

Toki, T., U. Tsunogai, T. Gamo, S. Kuramoto, and J. Ashi (2004), Detection of low-chloride fluids beneath a cold seep field on the Nankai accretionary wedge off Kumano, south of Japan, Earth and Planetary Science Letters, 228, 37-47.

Tsunogai, U. and H. Wakita (1995), Precursory Chemical Changes in Ground Water: Kobe Earthquake, Japan, Science, 269, 61-63.

Tsunogai, U., N. Yoshida, and T. Gamo (2002), Carbon isotopic evidence of methane oxidation through sulfate reduction in sediment beneath cold seep vents on the seafloor at Nankai Trough, Marine Geology, 187, 145-160.

Tunnicliffe, V., A.G. McArthur, and D. McHugh (1998), A Biogeographical Perspective of the Deep-Sea Hydrothermal Vent Fauna, Advances in Marine Biology, Vol. 34 (J.H.S. Blaxter, A.J. Southward, and P.A. Tyler eds.), Academic Press, 353-442.

Van Dover, C.L. (2000), Evolution and Biogeography, The Ecology of Deep-Sea Hydrothermal Vents, Prinston Univ. Press, 313-354. 